\title{
Optimized sleep strategy based on clustering in dense heterogeneous networks
}

\author{
Jun $\mathrm{Li}^{1,2^{*}}$, Hao Wang ${ }^{2}$, Xiumin Wang ${ }^{2}$ and Zhengquan $\mathrm{Li}^{1,3}$
}

\begin{abstract}
As a large number of small base stations have been deployed in dense heterogeneous networks, the increasing cost of energy has become a challenging issue. In this paper, we propose an optimized clustering-based sleep strategy to release the power consumption and interference in the system. First, we group the small base stations with large interference as a cluster in which the binary particle swarm optimization (BPSO) algorithm is used to formulate a sleep strategy for small base stations in the clusters. Since the small base stations between clusters have less interference, the whole spectrum resources can be shared in all clusters and the orthogonal spectrum resources are allocated for the small base stations in the same cluster. The strategy effectively restrains the co-tier interference in the system. Furthermore, a separate sleep strategy is applied for each cluster to improve the sleep and activation efficiency of the small base stations. The simulation results show that the proposed sleep strategy can improve the satisfaction of user equipment and reduce the power consumption. As the increasing number of user equipment, the outage probability of the system can be reduced effectively.
\end{abstract}

Keywords: Clustering, Heterogeneous networks, Outage probability, Power consumption, Sleep strategy

\section{Introduction}

With the huge demands of terminal equipment and development of the Internet, people put forward higher requirements of the data traffic usage. The traditional single-layer network has been unable to meet the users' quality of service (QoS) demands [1]. Small base station (BS) has the advantages of low transmission power, easy deployment, and maintenance. Therefore, the small BSs get more and more applications, which form a new heterogeneous network (HetNet) structure with the macro BSs [2-5].

The deployment of small BSs in HetNets can effectively reduce the loads of macro BSs and make the indoor users get a better QoS. With the increasing number of small BSs, a new dense heterogeneous network which exists severe co-tier interference and cross-tier interference is

\footnotetext{
* Correspondence: 07a0303105@cjlu.edu.cn

${ }^{1}$ National Mobile Communications Research Laboratory, Southeast University, Nanjing 210096, China

${ }^{2}$ Key Laboratory of Electromagnetic Wave Information Technology and

Metrology of Zhejiang Province, College of Information Engineering, China

Jiliang University, Hangzhou 310018, China

Full list of author information is available at the end of the article
}

formed [6-8]. Thus, how to reduce the existence of interference has become one of the current focuses. The authors in [9] presented a user-selected power control algorithm based on the non-cooperative game theory, which reduced the cross-layer interference of the system and improved the capacity of the system. A novel interference cancelation algorithm was proposed in [10] to mitigate the interference, which could be applied to different channel cases by applying channel estimation and eliminating the interference signal in the time domain. In [11], the authors employed a hybrid spectrum allocation method whereby the spectrum was split between the macro BSs and the nearby interfering small BSs based on resource demands, while the distant small BSs shared the entire spectrum. The authors in [12] quantified the impact of the user activity by comparing the performance of centralized resource-allocation schemes with that of a simple random access protocol.

In dense HetNets, the system consumes more power due to the deployment of a large number of small base stations [13]. In addition, the users have a certain degree of liquidity, especially for places of work and some 
large-scale activities. This phenomenon leads to the dynamic changes of loads in actual system. Typically, most of the BSs maintain a large power transmission to meet the satisfaction of the user equipment (UE), resulting in a larger system power consumption [14]. Therefore, studies about how to reduce the power consumption and saving unnecessary power waste become important. The authors in [15] presented an energy -efficient user association scheme based on the sleep mechanism of small BSs, which improved the energy efficiency of the network. In [16], an energy-saving small cell sleeping mechanism and enhanced inter-cell interference coordination techniques were proposed to decrease the energy consumption in HetNet. In order to improve the energy efficiency in HetNet, the authors in [17] put high-power macro BSs into sleep mode and offloaded the users to low-power small BSs or neighboring macro BSs. To release the additional overall energy consumption of the system, the work in [18] compared three dynamic algorithms to control a sleep mode of the low transmit power nodes (LPNs), in which most components in the LPN were shutdown. The authors in [19] considered both the partial spectrum reuse scheme and the base station sleep model and proposed a joint optimization method to improve the energy efficiency of the network. Assuming that both macro and small-cell base stations may be put into sleep mode, the authors in [20] formulated the optimization problem of resource partitioning and user association to minimize the total energy of the system. To improve the energy efficiency and system throughput of the system in the HetNets, a cell throughput based on sleep control scheme for the small base stations was proposed in [21]. To improve the energy efficiency of the whole system, the authors in [22] proposed an algorithm combining BS sleeping with dynamic clustering (SMDC) to maximize EE. A separation architecture called a hyper-cellular network $(\mathrm{HCN})$ was proposed in [23], which decoupled the control signals and data transmission functions. Moreover, switching off small cell base stations (BSs) or keeping them in energy-saving mode is a good way to improve EE and guarantee the quality of service (QoS) [24]. And an alternating iterative approach was proposed to solve the resulting multivariable optimization problem. In [25], the energy efficiency was optimized by sleep mechanism under average connection ratio (ACR) and average user rate constrains in the way of formulating an optimal maximum connection number of small cell to minimize the energy consumption subject to the joint constraint of ACR and average rate. Although such BSs are small-scale with lower power, the aggregated energy consumption of all BSs would be remarkable which results in the increased environmental and economic concerns. Turning off under-utilized BSs and maximizing the system energy efficiency will be a good way to solve the problem [26, 27].

At present, many studies attempt to propose different methods to release the interference and power consumption of BSs in the network separately. However, most of the schemes rarely consider the interference problem in dense heterogeneous networks, which leads to the decrease of performance in the network. Meanwhile, some of the system still has the certain power waste, which is contrary to the currently advocation of green communication. With the increasing number of small BSs in the dense HetNets, the interference and power consumption increase simultaneously. Therefore, we jointly consider the BS clustering and spectrum reuse to reduce the interference of the system. In addition, the BS sleeping strategy has been proposed in different clusters to reduce the power consumption of the system, which helps to improve the performance of the network.

In this paper, we take the clusters of the base station into consideration in the sleep strategy and propose an optimized clustering-based sleep strategy to reduce the interference of the network and decrease the power consumption of the system. We group the small BSs with large interference as a cluster to reduce the co-tier interference in the network. Thus, the small BSs between different clusters can share the whole spectrum resources, while the small BSs in the same cluster are allocated for the orthogonal spectrum resources. Then, a binary particle swarm optimization (BPSO) algorithm based on user association scheme is proposed to formulate a sleep strategy in the clusters to decrease the power consumption in the system.

The contributions of this paper are (1) small BSs between clusters share the whole spectrum due to the small interference, which enhances the system spectral efficiency; (2) the co-tier interference of HetNet is reduced through the method of grouping the small BSs as different clusters, and (3) the proposed sleep strategy reduces the outage probability of the system.

The rest of this paper is organized as follows: we introduce the system model of the dense HetNets in Section 2. In Section 3, an optimized clustering-based sleep strategy which includes the clustering stage and sleeping stage is proposed. Performance and simulation results are presented in Section 4. Section 5 gives the conclusion of this paper.

\section{System model}

\subsection{Network model}

We consider a downlink model of HetNet system as shown in Fig. 1. In our model, both of two tiers are considered as cellular network model which consists of radius $R_{m}$ for macro BSs and $R_{s}$ for outdoor small 


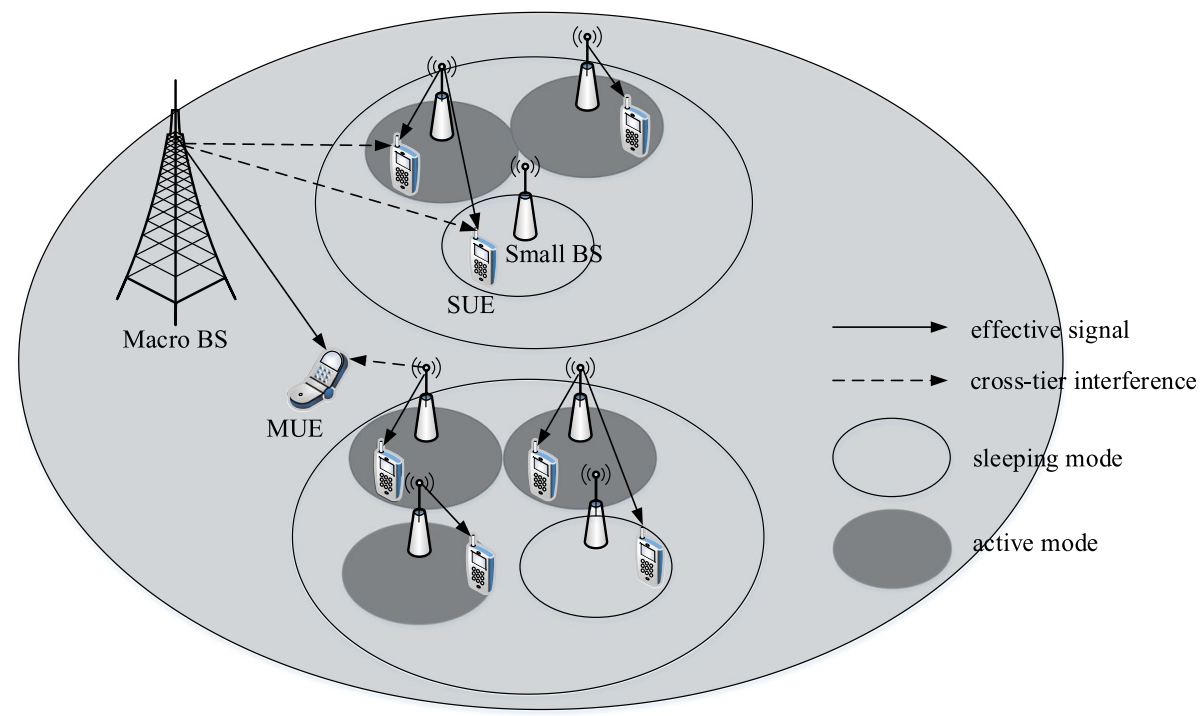

Fig. 1 HetNet system model. We consider a downlink model of HetNet system as shown in Fig. 1. In our model, both of two tiers are considered as cellular network model which consists of radius $R_{m}$ for macro BSs and $R_{s}$ for outdoor small BSs, such as macro cells and pico cells. We define the user equipment which connects to the macro BS and small BS as MUE and SUE, respectively. We assume that the UEs are uniformly distributed in the network, and each UE will associate with a macro BS or a small BS

BSs, such as macro cells and pico cells. We define the user equipment which connects to the macro BS and small BS as MUE and SUE, respectively. We assume that the UEs are uniformly distributed in the network and each UE will associate with a macro BS or a small BS.

In the coverage of the macro BSs, the small BSs are grouped as different clusters. Both macro BSs and small BSs which are in different clusters share the whole frequency band. The system bandwidth is $B$, and frequency reuse factor is one.

Assuming that the transmission power of small BS $i$ and macro BS $h$ are $P_{i}$ and $p_{h}$, respectively. Due to the outdoor scenario of the HetNet, the path loss between $\mathrm{BS}$ and $\mathrm{UE}$ is given by

$$
L=37+10 \log _{10}\left(d^{\eta}\right)+S
$$

where $d$ is the distance between BS and UE, $\eta$ is the path loss exponent, and $S$ is a zero-mean normal random variable that captures the random shadowing. Based on the path loss model, we can get the channel gain between BS and UE as

$$
G=10^{-L / 10}
$$

where $L$ is the path loss value.

Define the signal-to-interference-plus-noise ratio (SINR) of UE $n$ which connects to the small BS $k$ as

$$
\operatorname{SINR}_{k, n}=\frac{P_{k, n} G_{k, n}}{\sum_{h=1}^{M} p_{h, n} G_{h, n}+\sum_{i=1, i \neq k}^{N} x_{i, n} P_{i, n} G_{i, n}+\delta^{2}}
$$

where $P_{k, n}$ is the transmission power of small BS $k$ when it is associated with UE $n, G_{k, n}$ is the channel gain between small BS $k$ and UE $n, p_{h, n}$ is the transmission power of macro BS $h$ when it is associated with UE $n$, $G_{h, n}$ is the channel gain between macro BS $h$ and UE $n$, $P_{i, n}$ is the transmission power of small BS $i$ when it is associated with UE $n, G_{i, n}$ is the channel gain between small BS $i$ and UE $n, M$ is the number of macro BSs and $N$ is the number of small BSs, $\delta^{2}$ is the noise power, $x_{i, n}$ is the association relationship between UE $n$ and small BS $i$, and $x_{i, n}=1$ indicates that the UE $n$ associates with the small $\mathrm{BS} i$; on the contrary, $x_{i, n}=0$. Like the small $\mathrm{BS}$, in the macro BS $h$, the SINR of UE $m$ is

$$
\mathrm{SINR}_{h, m}=\frac{p_{h, m} G_{h, m}}{\sum_{l=1, l \neq h}^{M} p_{l, m} G_{l, m}+\sum_{i=1}^{N} x_{i, m} P_{i, m} G_{i, m}+\delta^{2}}
$$

\subsection{Power consumption model}

We adopt the power consumption model proposed in [28] which consists of two parts, i.e., static power consumption and dynamic power consumption. We use the linear approximation model to calculate the power consumption of small BSs, which is given as 


$$
P_{i, n}= \begin{cases}\mu P_{\mathrm{t}}+P_{\mathrm{c}} & \text { the small BS } i \text { is in active mode } \\ P_{\mathrm{s}} & \text { the small BS } i \text { is in sleeping mode }\end{cases}
$$

where $P_{i, n}$ is the transmission power of small BS $i$ when it is associated with UE $n, \mu$ is a coefficient associated with data traffic usage, $P_{t}$ denotes the transmission power of small BS, $P_{\mathrm{c}}$ represents the static power consumption of small BS in the active mode which is independent with transmission power, and $P_{s}$ is the small BS power consumption in the sleeping mode.

In order to evaluate the efficiency and performance of the system, we use the ratio of the number of outage users to the number of whole UEs in the system to represent the outage probability and it can be defined as

$$
P_{\mathrm{b}}\left(\operatorname{SINR}_{n}<\sigma\right)=\frac{N_{\mathrm{b}}}{N_{\mathrm{all}}}
$$

where $\operatorname{SINR}_{n}$ is the SINR of UE $n, \sigma$ is the minimum SINR requirement of the UEs, $N_{b}$ is the number of outage UEs, and $N_{\text {all }}$ is the number of the whole UEs. It is noted that the UE $n$ is assumed to be an outage UE when $\operatorname{SINR}_{n}<\sigma$.

\section{Optimized clustering-based sleep strategy}

\subsection{Clustering forming algorithm for small BSs}

In this section, we construct an interference graph $K$ $=(A, E, W)$, where $A=\left\{a_{1}, a_{2}, \ldots, a_{N}\right\}$ is the vertex set representing the set of small BSs and $N$ is the number of small BSs. Furthermore, $E=\left\{e_{1}, e_{2}, \ldots, e_{H}\right\}$ is the edge set, where $H$ is the number of edges in the graph, and each edge of the interference graph denotes the path loss values of small BSs in the network. $W=\left\{w_{1}, w_{2}, \ldots, w_{H}\right\}$ is the set of weights which corresponds to the vertexes. Note that the larger the sum of path loss of small BSs is the larger the weight of the interference is. And the weight of small BS $i$ can be expressed as

$$
w_{i}=\sum_{k} \lambda_{i k}
$$

where $\lambda_{i k}$ is the interference between small BS $i$ and neighboring small BS $k$ [29]. Due to the interference between small BSs can be evaluated by the interference between the BS and the UEs in the neighboring BS. Thus, we can calculate the interference between different small BSs according to the reference signal received power of the UEs, which can be expressed as

$$
\lambda_{i, k}=\max \left\{\max _{\forall n \in U_{i}}\left(P_{i, n} G_{i, n}\right), \max _{\forall n \in U_{k}}\left(P_{k, n} G_{k, n}\right)\right\}
$$

where $U_{i}$ and $U_{k}$ are the sets of UEs in the small BS $i$ and small $\mathrm{BS} k$, respectively.
We group the small BSs as different clusters based on their interference. First, we formulate the set of small BSs $i=\{1,2, \ldots, N\}$. We use $T$ as the interference threshold between the small BSs. The interference threshold $T$ is related to the number of users and BSs in the system. Referring to [16] and considering the system model of our paper and the actual situation of the system, it is reasonable that the threshold $T$ be set as $60-80 \%$ of the maximum interference value. The maximum number of small BSs in each cluster is $N_{\max }$.

Then, we consider the first small BS as the cluster head and calculate the weight of next small BS in the same cluster. We can add the small BS into the cluster when $w_{i}>T$ and mark the cluster.

During the cluster forming process, the interference values can be represented by the elements of weights. Note that if the interference value $w_{i}<T$ or the number in the cluster is larger than the threshold $N_{\max }$, we consider the small BS as the head in next cluster. The cluster forming algorithm for small BSs is denoted in Algorithm 1.

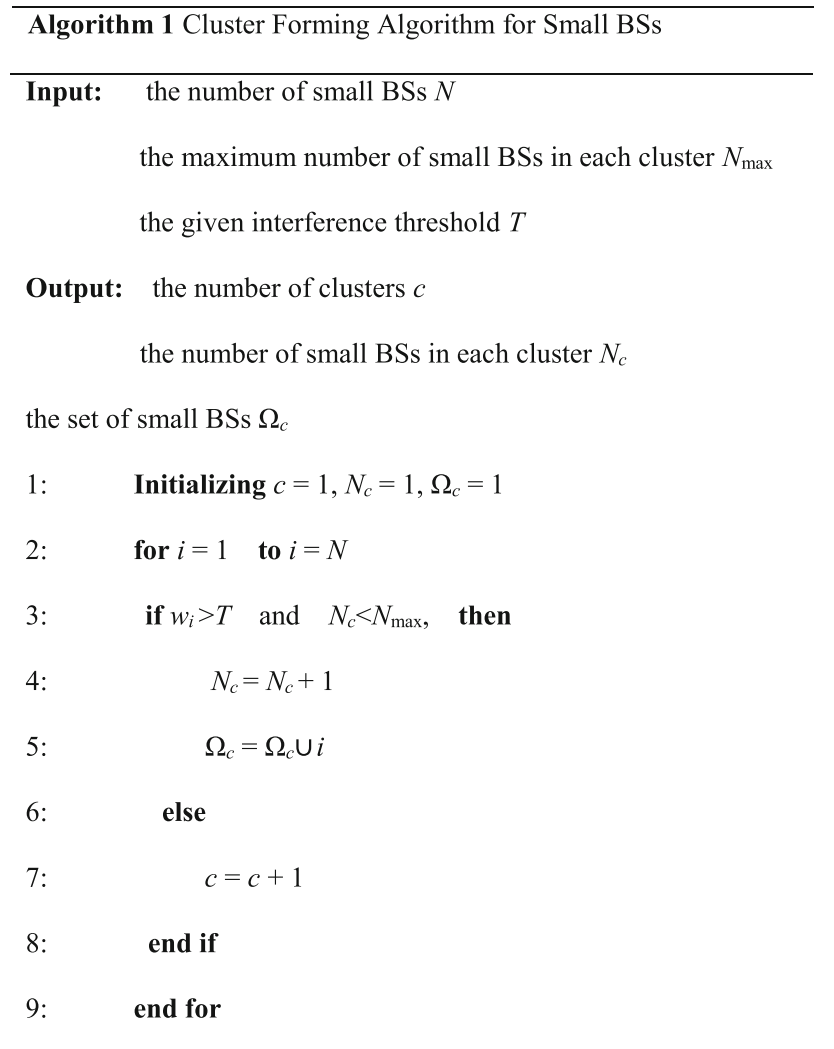

The system of the network can be clustered according to Algorithm 1 when the initial network is fixed. When the network is dynamic, we can determine if the new small BS can be added to the cluster according to the value of the interference and the number of small BSs in the cluster. If the interference is less than the threshold 
or the number of small BSs within the cluster is greater than $N_{\max }$, the small BS cannot add to the cluster. Therefore, the clustering algorithm adapts to the dynamic characteristic of the network.

\subsection{Sleeping algorithm in each cluster}

After the cluster forming procedure, the small BSs in the HetNets are grouped as $c$ clusters. Then, we propose a sleeping mechanism based on a binary particle swarm optimization (BPSO). In order to improve the SUEs' satisfaction and achieve the system load balancing, we use the SINR value of SUEs as the quality of service (QoS) of the system and consider the power consumption which is associated with traffic loads.

For the general communication system, we hope that the UEs in the system can obtain higher SINR value and consume lower power consumption. In such sense, we introduce the definition of overall weight which considers both of two parameters synthetically. The weight between the UE $n$, and the small BS $i$ is defined as

$$
q_{i, n}=\alpha \hat{\operatorname{SINR}}_{i, n}+\beta \hat{P_{i, n}}=\alpha \frac{\operatorname{SINR}_{i, n}}{\max \left(\operatorname{SINR}_{i, n}\right)}+\beta \frac{P_{i, n}}{\max \left(P_{i, n}\right)}
$$

where $\operatorname{SINR}_{i, n}$ is the normalized SINR value of UE $n, \hat{P}_{i, n}$ is the normalized load value of small BS $i, \mathrm{SINR}_{i, n}$ is the SINR of UE $n$ in the small BS $i$, and $P_{i, n}$ represents the power consumption of small BS $i$ which connects to the $\mathrm{UE} n . \alpha$ is the factor of QoS, and $\beta$ is the factor of power consumption in the system. The sum of $\alpha$ and $\beta$ is 1 . We can dynamically adjust the values of $\alpha$ and $\beta$ according to the weight of factors during the modeling process.

In the dense HetNets structure, we use the total weights of system to describe the overall performance of the network, which can be expressed as

$$
R=\sum_{i=1}^{N_{c}} \sum_{n=1}^{N_{u}} q_{i, n} x_{i, n}
$$

where $q_{i, n}$ is the weight of UE $n$ in the small BS $i, N_{c}$ is the number of small BSs in the cluster, and $N_{\mathrm{u}}$ is the number of UEs in the cluster.

Therefore, in order to consider the user satisfaction and power consumption of the system, we can formulate the problem of optimization model as

$$
\begin{aligned}
& \max _{x, P} \sum_{i=1}^{N_{c}} \sum_{n=1}^{N_{u}} q_{i, n} x_{i, n} \\
& \text { s.t. }\left\{\begin{array}{l}
0 \leq P_{i, n} \leq P_{\max } \\
\sum_{i=1}^{N_{c}} x_{i, n}=1 \\
x_{i, n}=\{0,1\} i=1,2, \ldots, N_{c}, n=1,2, \ldots, N
\end{array}\right.
\end{aligned}
$$

Due to the value of $x_{i, n}$ is 1 or 0 , such an optimization problem is NP-Hard. In order to speed up the optimization process and obtain a satisfying near-optimal solution, we propose a strategy which is based on a BPSO algorithm.

In the BPSO, each particle has certain position information and velocity information, which can be represented by UEs in the dense HetNets. The objective function value of the current position of the particle is represented by the total weight of the system. The position information of the particle can be used as the potential solution of the above optimization problem.

When each particle is used to find the optimal solution, two optimal positions are discussed, i.e., the individual optimal position and the global optimal position. We can calculate the individual optimal position of the $j$ th particle in the $j$ th iteration based on its total weight. The rule is shown as

$$
Z_{j}(t)= \begin{cases}X_{j}(t) & \text { if } R\left[X_{j}(t)\right]>R\left[Z_{j}(t)\right] \\ Z_{j}(t-1) & \text { if } R\left[X_{j}(t)\right] \leq R\left[Z_{j}(t)\right]\end{cases}
$$

where $Z_{j}(t)$ is the individual optimal position and $X_{j}(t)$ is the current position.

We can get the global optimal position in the th iteration based on the values of the individual optimal positions searched by the particle swarm, which can be expressed as

$$
C(t)=\arg \max _{1 \leq j \leq N_{c}}\left\{R\left[Z_{j}(t)\right]\right\}
$$

Then, the particles update their own velocity information and position information by comparing the individual optimal position to global optimal position, which can be denoted as

$$
\begin{aligned}
V_{j}(t+1)= & h(t) V_{j}(t)+c_{1} r_{1}\left(Z_{j}(t)-X_{j}(t)\right) \\
& +c_{2} r_{2}\left(C_{j}(t)-X_{j}(t)\right) \\
X_{j}(t+1)= & V_{j}(t+1)+X_{j}(t)
\end{aligned}
$$

where $h(t)$ represents the inertia weight in the $t$ th iteration, $c_{1}$ and $c_{2}$ are learning factors, and $r_{1}$ and $r_{2}$ are uniformly distributed random numbers. In order to ensure that the algorithm can achieve the convergence in the case of global search and local search, the inertia weight is linearly decreasing with time, which is expressed as

$$
h(t)=\frac{\left(h_{\mathrm{ini}}-h_{\mathrm{end}}\right)\left(D_{\max }-t\right)}{D_{\max }}+h_{\mathrm{end}}
$$

where $h_{\text {ini }}$ is the initial inertia weight and $h_{\text {end }}$ is the final inertia weight, and $D_{\max }$ is the maximum number of iteration. 
Thus, in a dense HetNet system, the process of the sleeping mechanism in each cluster can be represented by Algorithm 2.

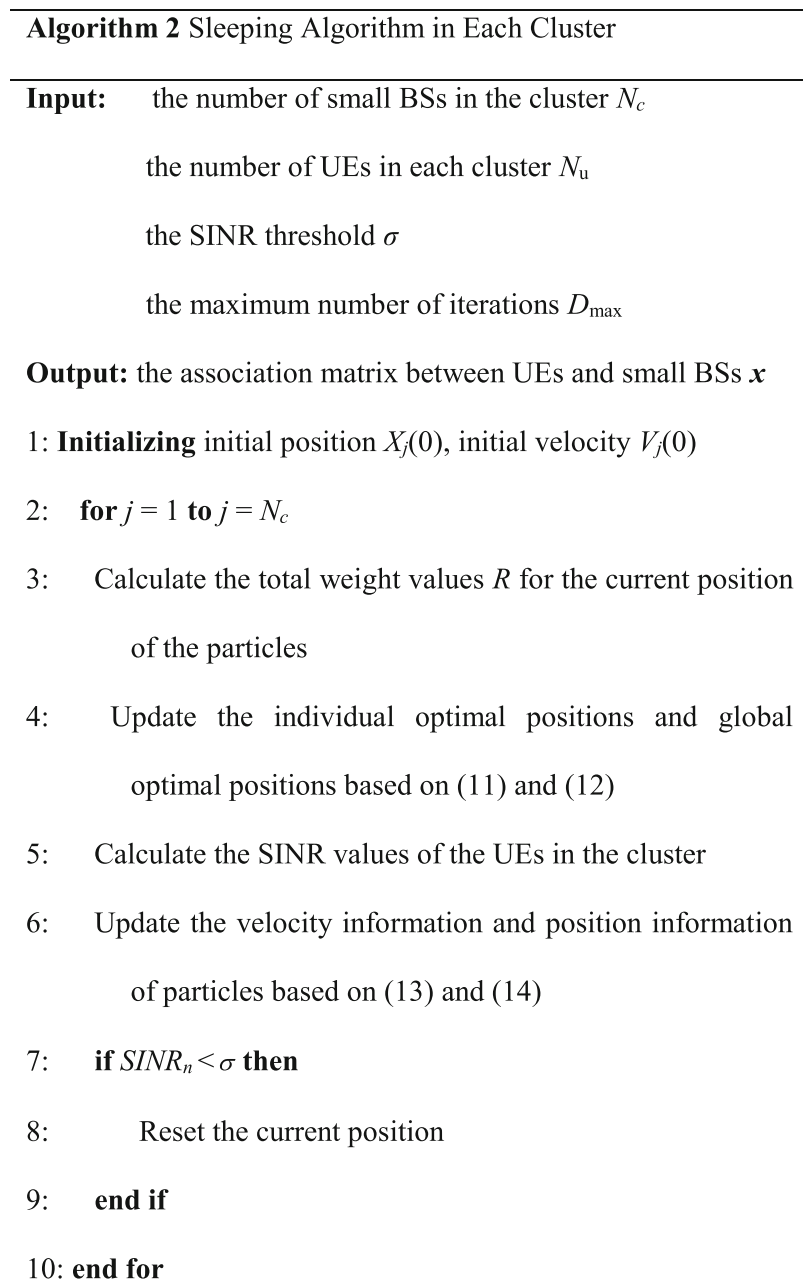

In the above optimized strategy which contains the clustering algorithm and sleeping algorithm, each UE calculates the total weight of the system for $N_{c}$ times in each iteration. $D_{\max }$ is the maximum number of iteration, and $c$ is the number of clusters. Assuming that the number of UEs in each cluster is $N_{u}$, the complexity of the strategy in the network can be expressed as $O\left(N_{c} N_{\mathrm{u}} c D_{\max }\right)$.

\section{Performance analysis}

This section focuses on the performance of the proposed strategy in the dense HetNets. We illustrate an example of combination of the macro BSs and the outdoor small BSs under 3GPP LTE-A HetNets framework, and we compare the proposed strategy to the general sleeping mechanism which is similar to [15]. The network performance of the SINR of
UEs, power consumption, and outage probability are analyzed. The simulation parameters are listed in Table 1. In our simulation, 1 macro BS is considered. In the macro BS, the small BSs are uniformly distributed. MUEs and SUEs are uniformly distributed in the coverage of macro BSs and small BSs, respectively. The number of the UEs in the HetNet is varying from low traffic load to high traffic load. It is noted that the traffic loads of UEs are different.

The cumulative distribution function (CDF) of the SUEs' SINR in the system is shown in Fig. 2. Since the use of the normal strategy which is similar to the one in [15] can improve the SINR performance of SUEs, the interference among small BSs is serious. The proposed strategy claims to share the whole spectrum between the clusters which effectively reduce the co-tier interference in the HetNet system. Therefore, it is more advantageous to improve the SINR performance of the SUEs.

Figure 3 depicts the power consumption of the system as the number of small BSs in the system increases. We can see that for the normal sleep strategy and no sleep strategy, the power consumption increases due to the co-tier interference in dense HetNets. Specially, the proposed strategy reduces the co-tier interference by clustering and includes the sleep mechanism. Thus, the strategy can effectively reduce the power consumption of the system.

In Fig. 4 and Fig. 5, we describe the impact of different values of $\alpha$ and $\beta$ on the network performance. In specific, the values of $\alpha$ and $\beta$ represent the weight of QoS and power consumption in the system. In Fig. 4, when the value of $\alpha$ is small, the SINR of UEs performs worse due to the lower weight of QoS. It is shown that the satisfaction of UEs improves when the value of $\alpha$ increases. In Fig. 5, we compare the power consumption of the system in

\begin{tabular}{lll}
\multicolumn{2}{l}{ Table 1 Simulation parameters } \\
\hline Symbol & Parameter & Value \\
\hline$R_{m}$ & Macro BS radius & $600 \mathrm{~m}$ \\
$R_{s}$ & Micro BS radius & $100 \mathrm{~m}$ \\
$p_{\max }$ & Maximum transmission power of macro BS & $46 \mathrm{dBm}$ \\
$P_{\max }$ & Maximum transmission power of micro BS & $20 \mathrm{dBm}$ \\
$\eta$ & Path loss exponent & $4[30]$ \\
$\delta^{2}$ & Noise power density & $-174 \mathrm{dBm} / \mathrm{Hz}$ \\
$B$ & System bandwidth & $10 \mathrm{MHz}$ \\
$C_{1}=c_{2}$ & Learning factors & 1.5 \\
$D_{\max }$ & Number of maximum iterations & 200 \\
\hline
\end{tabular}




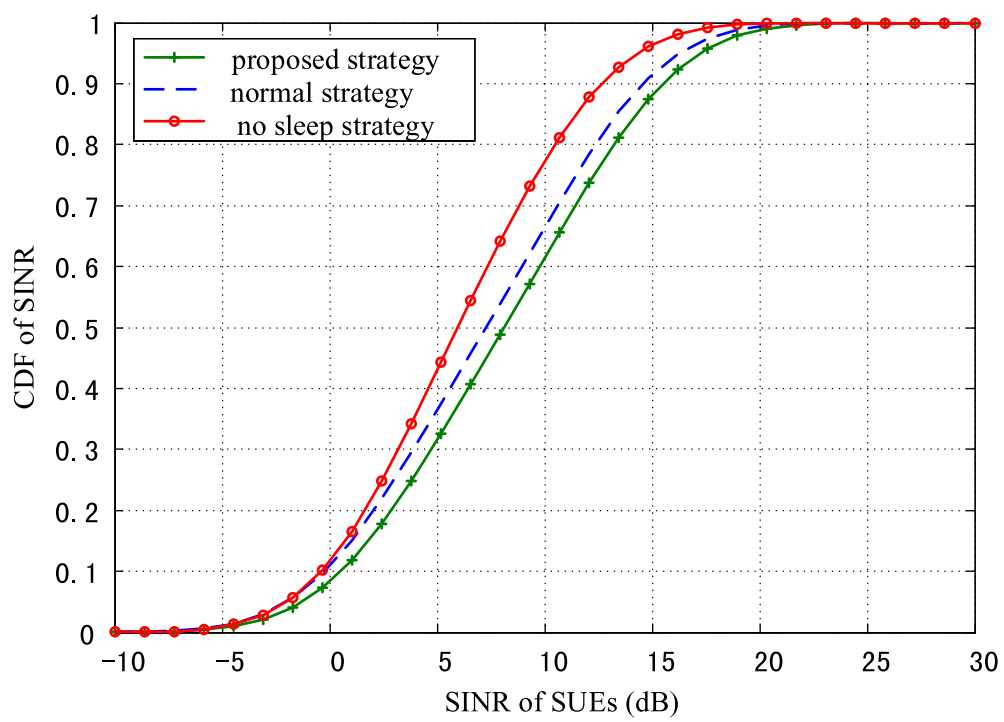

Fig. 2 SUEs' SINR CDF. The cumulative distribution function (CDF) of the SUEs' SINR in the system is shown in Fig. 2. Since the use of the normal strategy which is similar to the one in [15] can improve the SINR performance of SUEs, the interference among small BSs is serious. The proposed strategy claims to share the whole spectrum between the clusters which effectively reduce the co-tier interference in the HetNet system. Therefore, it is more advantageous to improve the SINR performance of the SUES

terms of $\beta$. It is observed that the power consumption of the system decreases because a larger $\beta$ implies a higher weight of the factor of power consumption. Therefore, we can set these two parameters to get a tradeoff between $\alpha$ and $\beta$ according to the requirement of the network.
The outage probability will be zero if there is no sleep mechanism in the system as shown in Fig. 6. It is possible that some small BSs enter the sleeping mode when the system takes the sleep strategy. It is shown that the outage probability increases firstly because the UEs' growth rate is larger than the

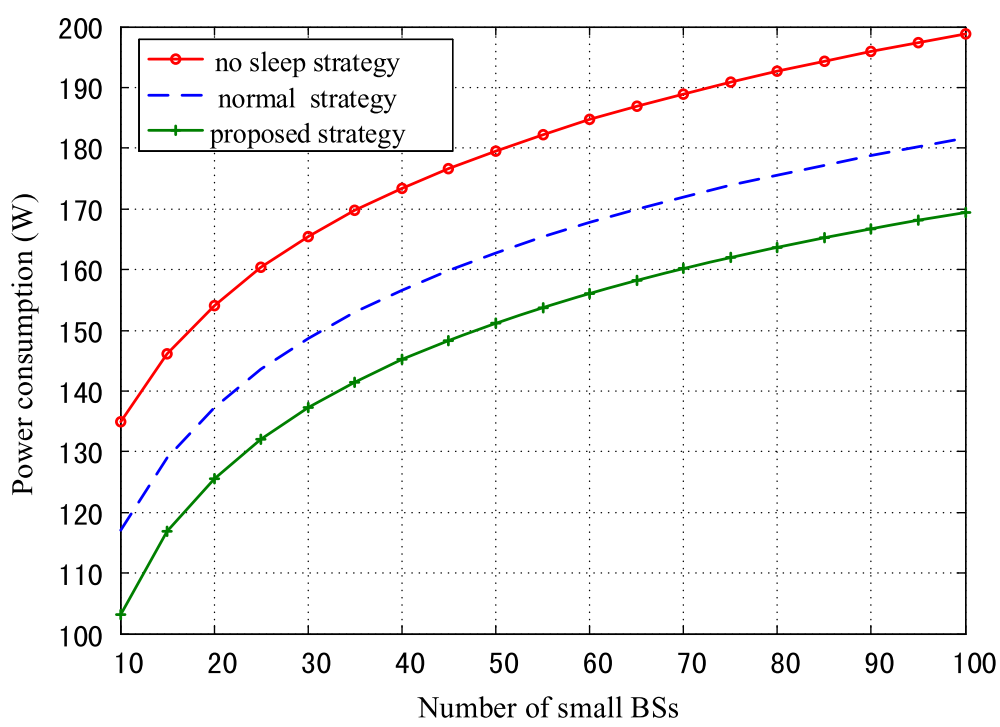

Fig. 3 Power consumption of the system. Figure 3 depicts the power consumption of the system as the number of small BSs in the system increases. We can see that for the normal sleep strategy and no sleep strategy, the power consumption increases due to the co-tier interference in dense HetNets. Specially, the proposed strategy reduces the co-tier interference by clustering and includes the sleep mechanism. Thus, the strategy can effectively reduce the power consumption of the system 


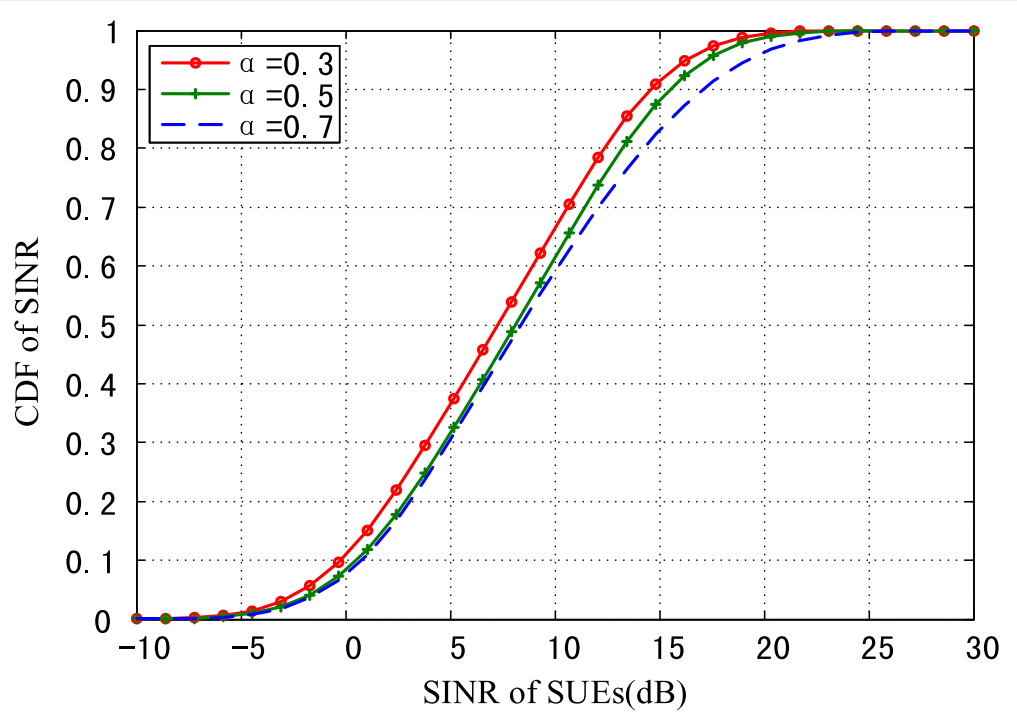

Fig. 4 Comparison of SINR performance with varying factor of $a$. In Fig. 4 and Fig. 5, we describe the impact of different values of $a$ and $\beta$ on the network performance. In specific, the values of $a$ and $\beta$ represent the weight of QoS and power consumption in the system. In Fig. 4, when the value of $a$ is small, the SINR of UEs performs worse due to the lower weight of QoS. It is shown that the satisfaction of UEs improves when the value of $a$ increases

activation rate. And the outage probability will gradually decline as the increasing number of activated small BSs. Therefore, the outage probability firstly increases and then decreases with the user's number. In the proposed strategy, we take the sleep mechanism in each cluster to improve the efficiency of sleep and activation. Hence, we can see the decline of outage probability in the system as a whole.

\section{Conclusion and discussion}

In this paper, we present an optimized clustering-based sleep strategy in the dense HetNets. We group the small BSs with large interference as a cluster based on the cluster forming algorithm. This helps reduce the co-tier interference and improve the satisfaction of UEs. Then, we take the sleep mechanism for small BSs in each cluster to improve the efficiency of sleeping and

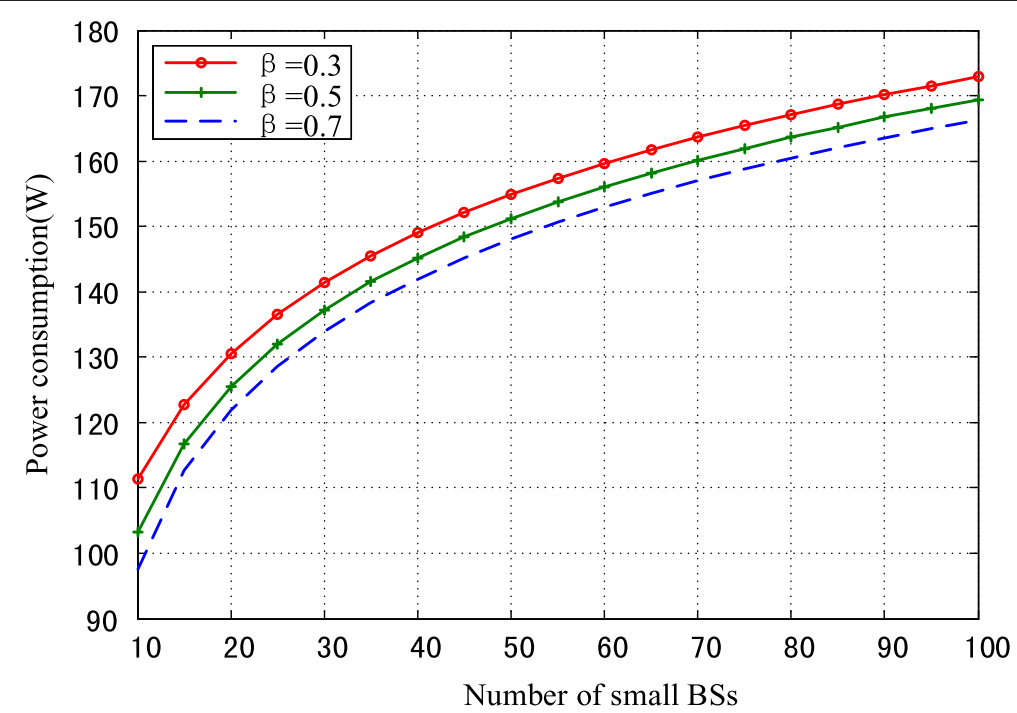

Fig. 5 Comparison of power consumption with varying factor of $\beta$. In Fig. 5, we compare the power consumption of the system in terms of $\beta$. It is observed that the power consumption of the system decreases because a larger $\beta$ implies a higher weight of the factor of power consumption. Therefore, we can set these two parameters to get a tradeoff between $\alpha$ and $\beta$ according to the requirement of the network 


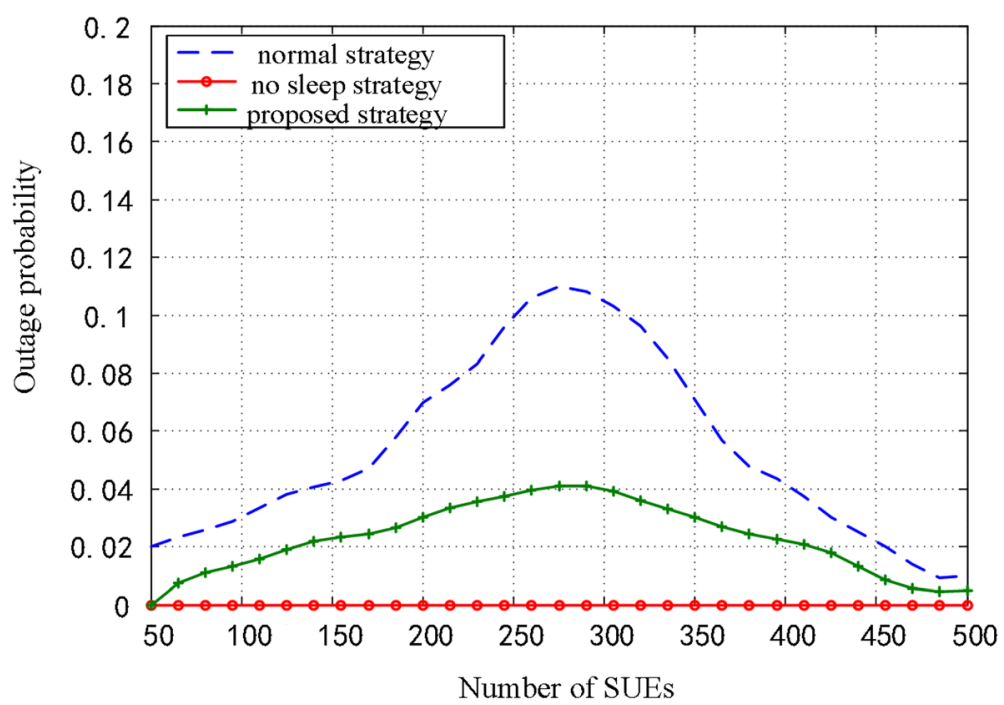

Fig. 6 Outage probability of the system. The outage probability will be zero if there is no sleep mechanism in the system as shown in Fig. 6 . It is possible that some small BSs enter the sleeping mode when the system takes the sleep strategy. It is shown that the outage probability increases firstly because the UEs' growth rate is larger than the activation rate. And the outage probability will gradually decline as the increasing number of activated small BSs. Therefore, the outage probability firstly increases and then decreases with the user's number. In the proposed strategy, we take the sleep mechanism in each cluster to improve the efficiency of sleep and activation. Hence, we can see the decline of outage probability in the system as a whole

activating operation. Furthermore, we change the values of the factor of QoS and power consumption to evaluate the performance of the system. The simulation results show that the SINR performance of the UEs is significantly improved by the proposed scheme compared with other strategies. Meanwhile, our method can perform much better in reducing the power consumption and the outage probability of the system.

\section{Abbreviation}

BPSO: Binary particle swarm optimization; BS: Base station; CDF: Cumulative distribution function; HetNet: Heterogeneous network; LPN: Low transmit power node; QoS: Quality of service; SINR: Signal-to-interference-plus-noise ratio; UE: User equipment

\section{Acknowledgements}

The authors are grateful for the valuable comments and suggestions provided by the reviewers.

\section{Funding}

This work was supported by the National Natural Science Foundation of China under grant nos. 61379027 and 61571108 and supported by Jiangsu Postdoctoral Foundation under grant no. 1302059B.

\section{Authors' contributions}

$J \mathrm{~L}$ and HW performed the experiments and wrote the paper, and XW and ZL helped to revise and improve the whole paper. All authors read and approved the final manuscript.

\section{Author information}

Jun Li was born in February, 1977. He received the Ph.D. degree in Communication and Information System from South China University of Technology, Guangzhou, China, in 2005. He worked in ZTE Corporation in 2005. And he has been an associate professor in China Jiliang University since 2007. His research interests include channel coding, signal detection, LTE physical layer standard, and wireless resource optimization.

Hao Wang was born in November, 1991. He received the B.S. degree from the Huaiyin Normal University in 2014. He is working toward the M.S. degree at China Jiliang University. His current research interest is in the area of the wireless resource optimization in heterogeneous network.

Xiumin Wang was born on April 1, 1963. She received the B.E. degree in Communication and Electronic System from Dalian University of Technology. She is now a professor and an Associate Dean of College of Information Engineering in China Jiliang University. Her research interests include signal and information processing.

Zhengquan Li received the B.S. degree from the Jilin University of Technology in 1998, the M.S. degree from the University of Shanghai for Science and Technology in 2000, and the Ph.D. degree in circuit and system from Shanghai Jiaotong University in 2003. He is currently a Professor with Jiangnan University. He is also a Postdoctoral researcher with the National Mobile Communications Research Laboratory, Southeast University. His current research interests include space time coding and cooperative communications and massive MIMO.

\section{Competing interests}

The authors declare that they have no competing interests.

\section{Publisher's Note}

Springer Nature remains neutral with regard to jurisdictional claims in published maps and institutional affiliations.

\section{Author details}

${ }^{1}$ National Mobile Communications Research Laboratory, Southeast University, Nanjing 210096, China. ${ }^{2}$ Key Laboratory of Electromagnetic Wave Information Technology and Metrology of Zhejiang Province, College of Information Engineering, China Jiliang University, Hangzhou 310018, China. ${ }^{3}$ State Key laboratory of Networking and Switching Technology, Beijing University of Posts and Telecommunication, Beijing 100876, China. 
Received: 1 March 2018 Accepted: 30 November 2018

Published online: 19 December 2018

\section{References}

1. Z.S. Zhang, K. Long, J.P. Wang, Self-organization paradigms and optimization approaches for cognitive radio technologies: a survey. IEEE Wirel. Commun. 20(2), 36-42 (2013)

2. V. Chandrasekhar, J.G. Andrews, A. Gatherer, Femtocell networks: a survey. IEEE Commun. Mag. 46(9), 59-67 (2008)

3. H.S. Dhillon, R.K. Ganti, F. Baccelli, J.G. Andrews, Modeling and analysis of Ktier downlink heterogeneous cellular networks. IEEE J. Select. Areas Commun. 30(3), 550-560 (2012)

4. A. Damnjanovic, J. Montojo, Y.B. Wei, T.F. Ji, T. Luo, M. Vajapeyam, T. Yoo, O. Song, D. Malladi, A survey on 3GPP heterogeneous networks. IEEE Wirel. Commun. 18(3), 10-21 (2011)

5. M.G. Peng, D. Liang, Y. Wei, J. Li, H.H. Chen, Self-configuration and selfoptimization in LTE-advanced heterogeneous networks. IEEE Commun. Mag. 51(5), 36-45 (2013)

6. C. Bouras, G. Kavourgias, V. Kokkinos, A. Papazois, in Proc. WTS, London, U. K. Interference management in LTE femtocell systems using an adaptive frequency reuse scheme (2012), pp. 1-7

7. F. Tariq, L.S. Dooley, A.S. Poulton, Y.S. Ji, in Proc. the 7th IWCMC, Wuhan, China. Dynamic fractional frequency reuse based hybrid resource management for femtocell networks (2011), pp. 272-277

8. D.L. Perez, I. Guvenc, G. Roche, M. Kountouris, T.Q.S. Quek, J. Zhang, Enhanced intercell interference coordination challenges in heterogeneous networks. IEEE Wirel. Commun. 18(3), 22-30 (2011)

9. X.M. Chai, X. Xu, Z.S. Zhang, A user-selected uplink power control algorithm in the two-tier femtocell network. Sci. CHN. Info. Sci. 58(4), $1-12(2015)$

10. H. Luo, W. Li, Y. Zhang, L.K. Huang, J. Cosmas, Q. Ni, CRS interference cancellation algorithm for heterogeneous network. Electro. Lett. 52(1), 77-79 (2016)

11. Y.L. Lee, J. Loo, T.C. Chuah, A.A. El-Saleh, Fair resource allocation with interference mitigation and resource reuse for LTE/LTE-A femtocell networks. IEEE Trans. Veh. Technol. 65(10), 8203-8217 (2016)

12. A. Mutairi, S. Roy, Impact of traffic load on OFDMA femtocells interference mitigation. IEEE J. Select. Areas Commun. 33(10), 2017-2026 (2015)

13. Y. Huang, X. Zhang, J.X. Zhang, J. Tang, Z.W. Su, W.B. Wang, Energy-efficient design in heterogeneous cellular networks based on large-scale user behavior constraints. IEEE Trans. Wirel. Commun. 13(9), 4746-4757 (2014)

14. K. Son, H. Kim, Y. Yi, B. Krishnamachari, Base station operation and user association mechanisms for energy-delay tradeoffs in green cellular networks. IEEE J. Select. Areas Commun. 29(8), 1525-1536 (2011)

15. Y.T. Zhu, Z.M. Zeng, T.K. Zhang, L. An, L. Xiao, in Proc. WPMC, Sydney, Australia. An energy efficient user association scheme based on cell sleeping in LTE heterogeneous networks (2014), pp. 75-79

16. R. Tao, J. Zhang, X.L. Chu, in Proc. IEEE 83rd Veh. Technol. Conf. An energy saving small cell sleeping mechanism with cell expansion in heterogeneous networks (VTC Spring, Nanjing, 2016), pp. 1-5

17. P. Ren, M.X. Tao, A decentralized sleep mechanism in heterogeneous cellular networks with QoS constraints. IEEE Wireless Commun. Lett. 3(5) 509-512 (2014)

18. L. Falconetti, L. Hevizi, I. Godor, in Proc. the 10th ISWCS, IImenau, Germany. Sleep mode control for low power nodes in heterogeneous networks (2013), pp. 1-5

19. X.M. Chai, Z.S. Zhang, K.P. Long, Joint spectrum-sharing and base station sleep model for improving energy efficiency of heterogeneous networks. IEEE Syst. J., vol. PP 99, 1-11 (2015)

20. C.L. Jia, T.J. Lim, Resource partitioning and user association with sleep-mode base stations in heterogeneous cellular networks. IEEE Trans. Wirel. Commun. 14(7), 3780-3793 (2015)

21. P. Phaiwitthayaphorn et al., in Proc. the 14th ECTI-CON, Phuket, Thailand. Cell throughput based sleep control scheme for heterogeneous cellular networks (2017), pp. 584-587

22. Y. Li, Y. F. Ma, Y. W and W. L. Zhao, Base station sleeping with dynamical clustering strategy of COMP in LTE-advanced in Proc. 2013 IEEE Internationa Conference on Green Computing and Communications and IEEE Internet of Things and IEEE Cyber, Physical and Social Computing. Beijing, China, pp. 157-162. 2013
23. S. Zhang, J. Gong, S. Zhou, Z.S. Niu, How many small cells can be turned off via vertical offloading under a separation architecture? IEEE Trans. Wirel. Commun. 14(10), 5440-5453 (2015)

24. C. Liu, B. Natarajan, H. Xia, in IEEE Transactions on Vehicular Technology, Vol. 65, No. 3. Small cell base station sleep strategies for energy efficiency (2016), pp. 1652-1661

25. Z. Pan, Q. Zhu, in IEEE Communications Letters, vol. 21, no. 5. Energy efficiency optimization in 3-D small cell networks-based sleep strategy (2017), pp. 1131-1134

26. M.J. Feng, S.W. Mao, T. Tao, BOOST: base station on-off switching strategy for green massive MIMO HetNets. IEEE Trans. Wirel. Commun. 16(3), 7319$7332(2017)$

27. M.J. Feng, S.W. Maom, T. Jiang, Base station ON-OFF switching in $5 \mathrm{G}$ wireless networks: approaches and challenges. IEEE Trans. Wirel. Commun. 24(3), 46-54 (2017)

28. C. Desset et al., in Proc. IEEE WCNC, Paris, France. Flexible power modeling of LTE base stations (2012), pp. 2858-2862

29. L.G.U. Garcia, G.W.O. Costa, A.F. Cattoni, K.I. Pedersen, P.E. Mogensen, in Proc. IEEE GLOBECOM, Miami, USA. Self-organizing coalitions for conflict evaluation and resolution in femtocells (2010), pp. 1-6

30. V. Chandrasekhar, J.G. Andrews, T. Muharemovic, Z.K. Shen, A. Gatherer, Power control in two-tier femtocell networks. IEEE Trans. Wirel. Commun. 8(8), 4316-4328 (2009)

\section{Submit your manuscript to a SpringerOpen ${ }^{\circ}$ journal and benefit from:}

- Convenient online submission

- Rigorous peer review

- Open access: articles freely available online

- High visibility within the field

- Retaining the copyright to your article

Submit your next manuscript at $\boldsymbol{\nabla}$ springeropen.com 